\title{
The Influence of Selenium Nanoparticles (NP) on Gut Health and Performance
}

\author{
Sheeana Gangadoo ${ }^{1}$, Rob Moore ${ }^{2}$, Robert Hughes ${ }^{3}$, Dragana Stanley ${ }^{1}$, James Chapman ${ }^{1}$ \\ ${ }^{1}$ Central Queensland University \\ Rockhampton, Australia \\ S.Gangadoo@cqu.edu.au; D.Stanley@cqu.edu.au; J.Chapman@cqu.edu.au \\ ${ }^{2}$ Royal Melbourne Institute of Technology \\ Melbourne, Australia \\ Rob.Moore@rmit.edu.au \\ ${ }^{3}$ South Australian Research and Development Institute \\ South Australia, Australia \\ Bob.Hughes@sa.gov.au
}

\section{Extended Abstract}

The innovative field of nanotechnology has rapidly integrated into multiple research areas including, but not limited to, medicine, electronics, transportation, environmental management, material and food applications. The chemical and physical properties of nano-scaled objects differ significantly from their bulk counterparts and therefore behave differently. Nano- size can offer the benefits of increased surface area to volume ratio, enhanced biocompatibility and efficient delivery of a substance to the body. In the poultry industry, nanoparticles of various materials, such as metallic, polymeric and other origins, are being investigated for various differing modes of action. They can be used as 1) antimicrobial agents, reducing the abundance of zoonotic human pathogens and enhancing the microflora, 2) growth promoters, enhancing expression of muscular growth factors, 3) immunity enhancers by increasing levels of antioxidant function, 4) drug and vaccine delivery and 5) improving overall performance and health of poultry. Opposing studies have shown that nanoparticles can also have detrimental effects; bioaccumulative effects, increased toxicity with permeability through the blood-brain barrier and gut dysbiosis, the perturbation of the normal microflora. The interaction effect of nanoparticles with the biological system needs to be thoroughly investigated as this seems to be the key for its successful application in a body [1].

In this study, nanoparticle of selenium (Se) was synthesised through chemical reduction. Selenium tetrachloride was reduced with ascorbic acid, where the selenium ions were reduced to form clusters. These clusters aggregate as their surface energy increased and grow in size. Poly(sodium 4-styrenesulfonate) was added as a protecting agent, forming an electrical bilayer around the nanoparticle and slowing down the growth of the clusters. This step is important as the occurring repulsion force from the capping agent is required to control particle size. Particle size of a nanoparticle can determine its entry, absorption and elimination properties throughout the body and is a crucial factor when utilised in a biological system. A full characterisation was performed on the selenium nanoparticle including the use of UV-vis and Elemental Dispersive X-ray (EDX) spectroscopy to confirm the presence of elemental selenium. Scanning Electron Microscopy (SEM) and Transmission Electron Microscopy (TEM) were used to visualise the nanoparticle and assess shape and morphology. The size of the Se-NP was evaluated using Dynamic Light Scattering (DLS) and Image J counting functions and crystallinity was performed using X-ray Diffraction [2].

The selenium nanoparticles were utilised as supplementation in broiler feed at different concentrations against two controls, inorganic and organic Se, currently used in the poultry industry. The results showed Se-NP did not affect bird weight or performance, but altered the gut microbiota of the birds, increasing both health-promoting and pathogenic strains. The concentration of SeNP was highly correlated with the abundance of Faecalibacterium, which is known to promote epithelial health and butyrate production. The results demonstrated best results with an intermediate concentration of Se-NP, $0.9 \mathrm{mg} / \mathrm{kg}$, showing improved gut health, enhanced short-chain fatty acids production and comparable weight gain [3]. 


\section{References}

[1] S. Gangadoo, D. Stanley, R. J. Hughes, R. J. Moore and J. Chapman, "Nanoparticles in feed: Progress and prospects in poultry research," Trends Food Sci Technol., vol. 58, pp. 115-126, 2016.

[2] S. Gangadoo, D. Stanley, R. J. Hughes, R. J. Moore and J. Chapman, "The synthesis and characterisation of highly stable and reproducible selenium nanoparticles," Inorganic and Nano-Metal Chemistry, vol. 47, no. 11, pp. 15681576,2017

[3] S. Gangadoo, I. Dinev, J. Chapman, R. J. Hughes, T. T. H. Van, R. J. Moore and D. Stanley, "Selenium nanoparticles in poultry feed modify gut microbiota and increase abundance of Faecalibacterium prausnitzii," Appl Microbiol Biotechnol., vol. 102, no. 3, pp. 1455-1466, 2018. 